\title{
Noise Characterization in Web Cameras using Independent Component Analysis
}

\author{
M.A.U. Khan, T.M. Khan, R.B. Khan, A. Kiyani, M.A. Khan
}

\author{
Mohammad Asmat Ullah Khan \\ Department of Electrical and Computer Engineering \\ Effat University Jeddah, Saudi Arabia. \\ mohammad_a_khan@yahoo.com \\ Tariq Mahmood Khan, \\ Muhammad Aurangzeb Khan \\ Department of Electrical Engineering \\ COMSATS Institute of Information Technology, \\ Islamabad, Pakistan. \\ tariq_mehmood@comsats.edu.pk,rabiya@ciit.net.pk
}

\author{
Rabia Bahadar Khan, Atiqa Kiyani \\ Department of Electrical Engineering \\ COMSATS Institute of Information Technology, \\ Abbotabad, Pakistan. \\ rabiya@ciit.net.pk,atiqa@ciit.net.pk
}

\begin{abstract}
An image captured by a web camera contains stationary and nonstationary noise patterns. These noise patterns are of three types i.e. Fixed Pattern Noise (FPN), Interactive Nose (IN) and Temporal Noise (TN). TN is an independent noise pattern and needs an algorithm that does exploit its higher-order dependencies. Previously, these noise patterns have been characterized using Principal Component Analysis (PCA). PCA is restricted to second order dependencies. In this paper Independent Component Analysis (ICA) has been investigated for actual TN noise. The experimental results demonstrates the effectiveness of the proposed method.
\end{abstract}

Keywords: fixed pattern noise, interaction noise, temporal noise, independent component analysis, principle component analysis.

\section{Introduction}

Web camera is a real-time device. It describes low-resolution digital video which is used for instant messaging or a PC video calling application. Just like any other electronic device, webcam's acquired images contain noise patterns. To be more specific, it can be claimed that a webcam image contains stationary and non-stationary noise patterns. Generally, the noise patterns are classified as Fixed Pattern noise (FPN), Temporal noise (TN), and Interaction noise (IN) [1].

The first noise pattern FPN is due to a combination of variations in image pixel geometry. It is observed that Fixed pattern noise emerge in very long exposures and is worsen by higher temperatures. Whereas, the second pattern Temporal noise (TN) fluctuates randomly from frame to frame and is characterized by intensity and color fluctuations near the actual image intensity. $\mathrm{TN}$ is present at any exposure length. The third noise pattern is referred to as Interaction Noise (IN), which is highly camera-dependent. IN is normally introduced by the camera when it reads data from the digital sensor. This type of noise is more visible in the shadows, or when an image has been excessively brightened. 
There has been a considerable research literature available to discuss ways and means for identification of these noise patterns. One common and popular method recently introduced for identification of these noise patterns is based on Principle Component Analysis (PCA) [2]. PCA in general, is statistical method to find useful image representations. Any given image can be decomposed into its combination of the standard basis images. The main objective in PCA is to find an optimum set of base images to uncorrelate the image coordinates into PCA coefficients which cannot be linearly found from each other. It has been an established fact that PCA can only extract and exploit pair wise linear dependencies between pixels. In the joint distribution of PCA coefficients the high-order dependencies are still there. It has already been confirmed that the higher order relationship among the pixels contains most of the important information of an image [3]. Later on, a generalization of PCA, known as Independent Component Analysis (ICA) has indeed provided better recognition rate as compared to PCA when used on Face Recognition data [4]. Motivated by the ICA success we started investigating web camera images. PCA representation of web camera noise characteristics may not be able to capture adequately the high-order structure present in the image. Specifically the object independent noise pattern TN identification is in need of an algorithm that does exploit higher-order dependencies. ICA is one such generalization of PCA that exactly fits the bill.

ICA seeks a set of independent components instead of a set of orthogonal components. Two components are considered as an independent components if we have knowledge about one and don't know any thing about the other. This is a very strong condition than uncorrelated components. Normally, we call ICA a blind source separation. Since, we have to find out a set of original sources from an observed mixture in blind source separation problem.

This paper presents the conjecture that ICA, a generalization of PCA, provides a better identification of noise patterns. For the purpose of practical implementation of ICA, numerous methods are available. The algorithm employed in this research word is developed by [5]. This algorithm has proven its worth by separating randomly mixed auditory signals (the cocktail party problem), and for separating electroencephalogram (EEG) signals [6] and functional magnetic resonance imaging (FMRI) signals [7].

This paper is organized as follows. In Section II, different types of webcam noises has been classified. In Section III, the conventional methods used for characterization of noises in webcams have been introduced. In Section IV, proposed work will be explained and the claim that ICA performs better than PCA will be elaborated further. In section V, results will be provided with discussion. Conclusion will be presented in the last section.

\section{Noise Characterization}

Electronic devices have some degree of noise when they receive or transmit data as a 'signal'. For television this signal is broadcast data; for webcams this signal is light which hits the camera sensors. The most common type of noises in webcams are:

1. Fixed pattern noise (FPN) is used to represent a noise pattern which is observed during longer exposure shots. It is a fixed pixel-to-pixel offset which is formed because of the combination of variations in image substrate material, pixel geometry and dark current as shown in Fig. 1.

2. Temporal noise (TN) sets the fundamental limit on image sensor performance, it is usually much more difficult to remove without degrading the image. Computers have a difficult time discerning $\mathrm{TN}$ from fine texture patterns such as those occurring in dirt or foliage, so removal of TN results in the loss of these textures as well, which is shown in Fig. 2. 


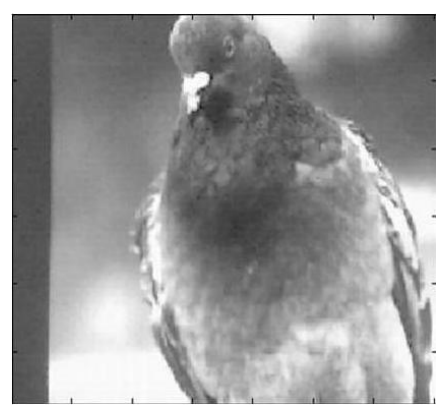

(a)

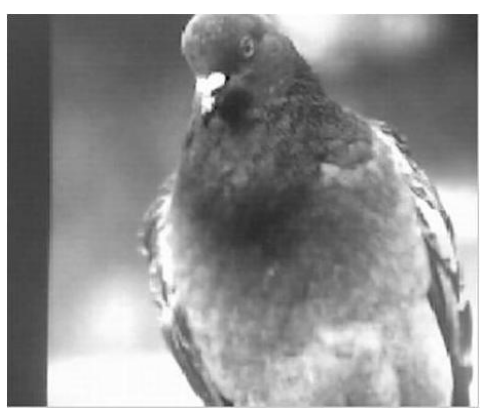

(b)

Figure 1: a) A sample image b) FPN extracted from a).

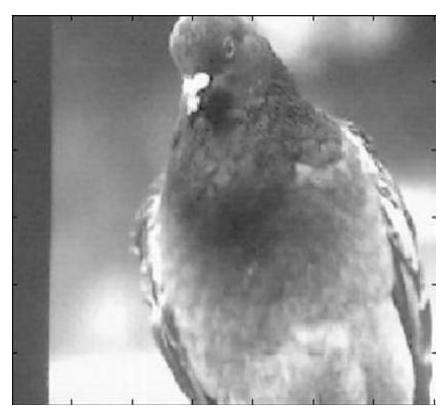

(a)

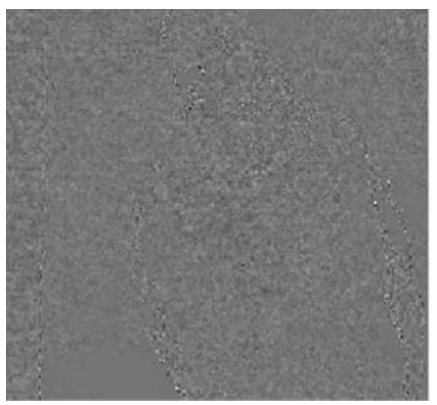

(b)

Figure 2: a) A sample image b) TN extracted from a).

3. Interaction noise (IN) is highly camera-dependent, and is introduced by the camera when it reads data from the digital sensor. It is present at those locations in the image where the signal varies the most as shown in Fig. 3.

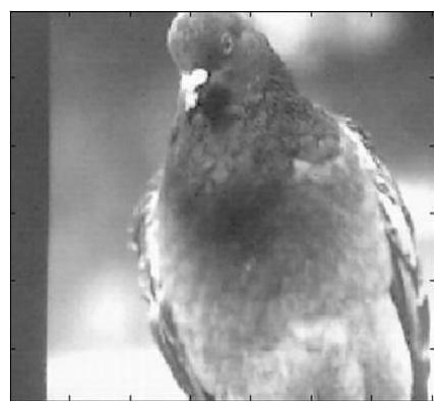

(a)

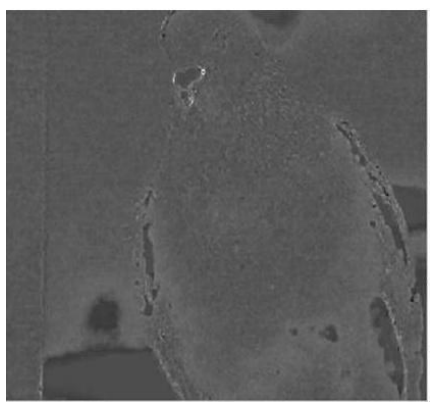

(b)

Figure 3: a). A sample image b) IN extracted from a).

Separating these from each other helps to improve image quality as it either educe or eliminate some noise components. 


\subsection{Working with PCA}

In this section, the salient features of PCA are elaborated. PCA uses Gaussian source models. $\mathrm{PCA}$ is a linear combination of basis vector. Let $\mathrm{X}$ be the original $n \times n$ matrix, $\mathrm{T}$ be the transformation matrix, $\mathrm{Y}$ would be the projection of the original matrix. As described in the following equation:

$$
Y=T X
$$

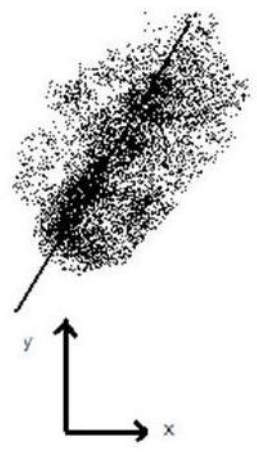

(a)

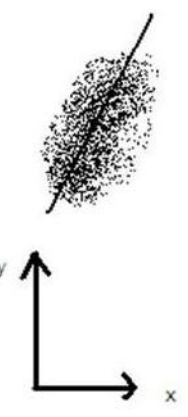

(b)

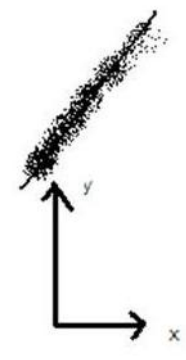

(c)

Figure 4: In a),b) and c) the best curve fitting line $\mathrm{Y}=\mathrm{TX}$ is shown by dark line.

The probability of the data depends only on first and second-order statistics if we have Gaussian source. In PCA, the rows (M) represents the eigenvectors of the covariance matrix of the data [1].

\subsection{Working with ICA}

It is inadequate when the actual sources are non-Gaussian and we assume a Gaussian sources in PCA. Signals can be described well as linear combinations of sources with long tailed distributions [5]. In such cases, ICA has the following advantages over PCA:

1. It uniquely identifies the mixing matrix M.

2. It provides a better probabilistic model of the data.

3. It finds non-orthogonal basis which are able to reconstruct the data better than PCA even in the presence of noise.

4. It is sensitive to high-order statistics in the data, and not restricted to second order statistics only.

ICA can be implemented by taking $\mathrm{X}$ transpose (n-dimensional data vector) and organize the given data so that images are in the columns of $\mathrm{X}$. This approach is illustrated in Fig. 4. In this approach, pixels are random variables and images are realizations. Here, the emphasis is on the independence of pixels or functions of pixels. For example, we have to consider pixel $i$ and $j$ independent if we are moving across the entire set of images. As, we can not predict the value taken by a specific pixel based on the corresponding value taken by pixel on the same image. This approach was inspired by [6] work on the ICs of natural images. 


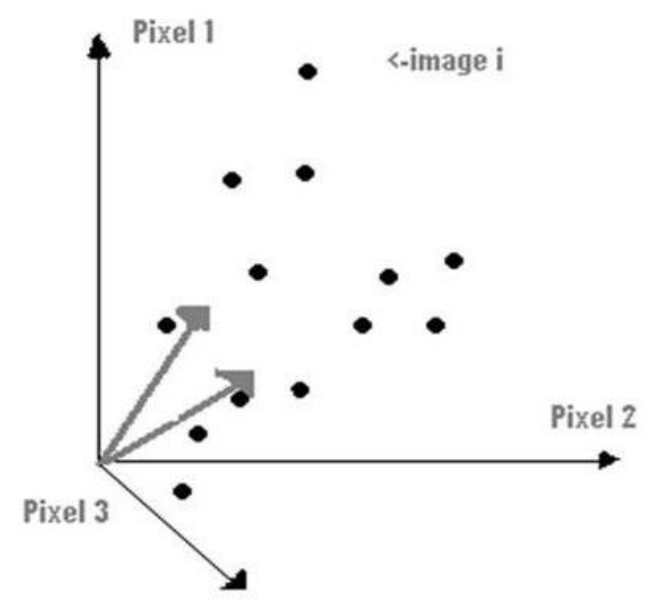

Figure 5: Finds weight vectors in the direction of statistical dependencies among the different images

\section{Conventional method}

In [1] the PCA method is applied to characterize the artifacts in fully digital image-acquisition systems. For practical application a video sequences was taken with USB cameras. Normally PCA method provides three different sets of spatial-temporal structures Fig. 6. Then a series of quasi sinusoidal patterns with different spatial frequencies were presented to the camera. With this approach, it is possible to characterize the noise using eigen-vector analysis.

In [2], Digital image noise has been characterized on the basis of RAW data. Noise characterization is done by shooting several frames both in complete darkness with several exposure times and also on several exposure levels with fixed field target. Based on these results, a number of parameters describing the camera's noise characteristics and sensitivity are measured.

\section{Proposed Method}

In digital cameras, there are three types of noises as discussed earlier. IN and TN are corelated to each other. PCA can be used efficiently to characterize these noises, but it is not able to provide adequate representation for the IN noise. One possible explanation that can be described for this failure is the inherent characteristic associated with IN noise. This unique characteristic is independence that PCA ignores but ICA does take into account.

For the ICA implementation for noise characterization of web camera, the setup has been laid out as following. A commercial web camera was connected directly to the computer via USB port. The camera provides frames of $288 \times 352$ pixels at a frame rate of $50 \mathrm{images} / \mathrm{s}$. The output values from the actual pixels in the field circuitry of the web camera are different from the values of the pixels in the analyzed set of frames. As artifacts are added by the compression algorithms and readout electronics in the analyzed pixels [1]. 


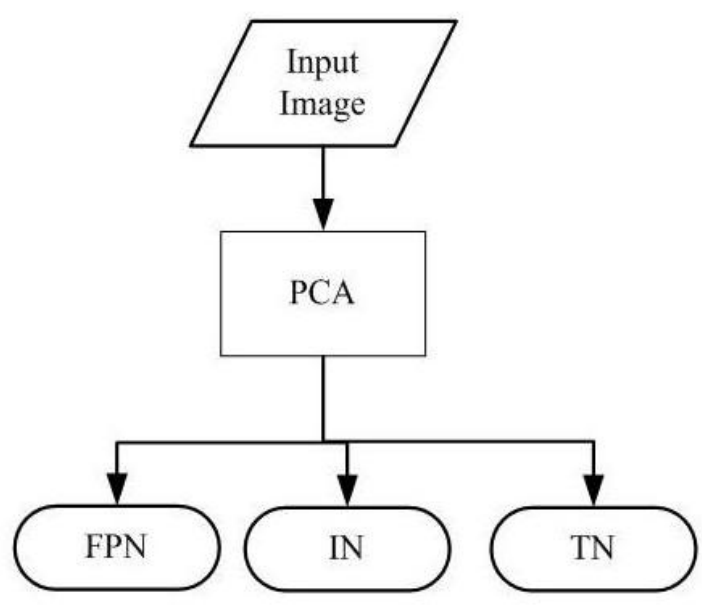

Figure 6: Block diagram of Conventional Method

The object is presented to the camera by using a CRT monitor. The web camera stares at the monitor and a movie is recorded to identify and classify the spatial-temporal artifacts embedded in the image. Each sequence contains 50 frames. The patterns spatial frequency varies slightly from the top to the bottom of the frame in order to include a narrow variation of the spatial frequency when moving along the vertical direction. Its basic aim is to preclude aliasing artifacts along the whole image. The experiment has been performed on three different frequencies to show how noise pattern changes with increasing frequencies. These three chosen frequencies are labeled as low, medium, and high frequencies are shown in Fig. 7.

The images are given as an input signal to PCA. It has already been observed that PCA is not suitable for separating independent noise processes. Therefore, PCA is used as helping tool to obtain two types of noise patterns i.e; FPN and non-FPN noise patterns. The non FPN noise patterns contain IN and TN, both are completely independent of each other. ICA is used to separate these two noise patterns.

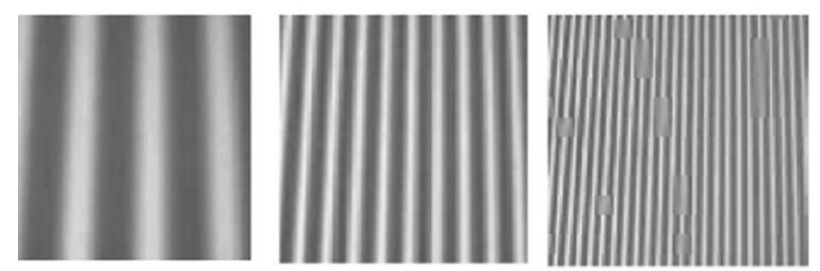

Figure 7: a) Low frequency frame, b) Medium frequency frame, c) high frequency frame.

ICA computes independent components and reconstruction is done by projecting these independent components. The process is demonstrated in Fig. 8. 


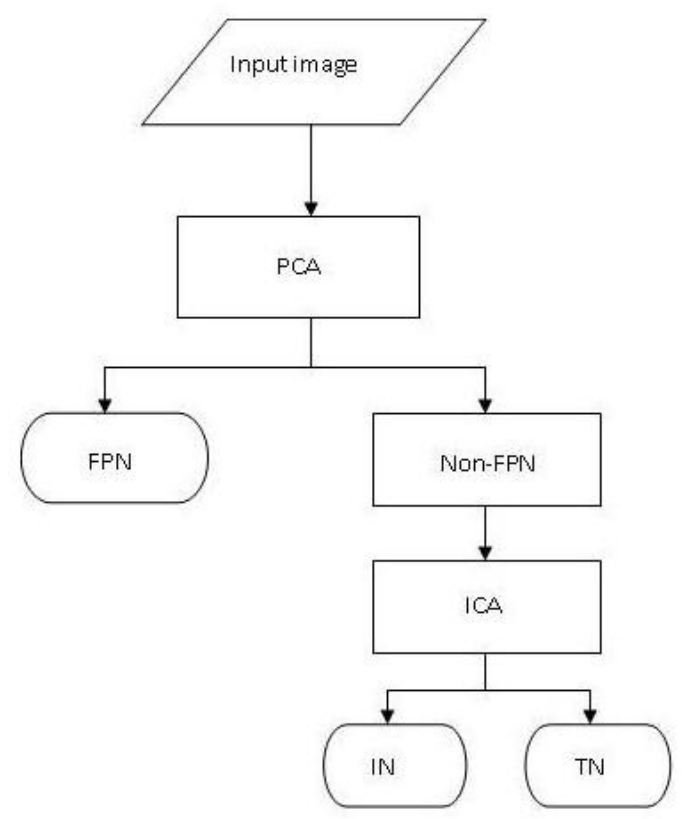

Figure 8: Block diagram of Proposed Method.

\section{$5 \quad$ Results and Discussions}

In the Fig. 9 it can be seen that FPN carries the maximum of variation of data. IN expresses less variation compare to TN. As PCA focuses on covariance structure thus gives good results for IN but it fails while discussing about TN noises, because TN is statistically independent noise.

As a solution to above problem, PCA has been applied first to the webcam images from which FPN and non FPN noises have been extracted. The non FPN (IN and TN) noise images are than processed further by the ICA. As they are statistically independent of each other therefore, ICA is performed on these images. The outputs of IN and TN noise images are shown in Fig. 10.

A pure $\mathrm{TN}$ is a diagonal covariance-wise for noises as it does not depend on the spatial frequency structure of the object. Comparing Fiq. 11 it can be observed that ICA outperforms PCA giving a pure diagonal. The diagonals using ICA from the three different frequencies are closer to the delta function than those retrieved using PCA.

The structure of the covariance of TN filtered set is strongly dependent on the spatial frequency. Therefore, it is better revealed when plotting the correlation function as an image. TN is a Gaussian noise pattern that is completely independent, thus when ICA is applied for different frequencies for extracting TN the results are more explicit than that attained by PCA as described in Fig. 12. 


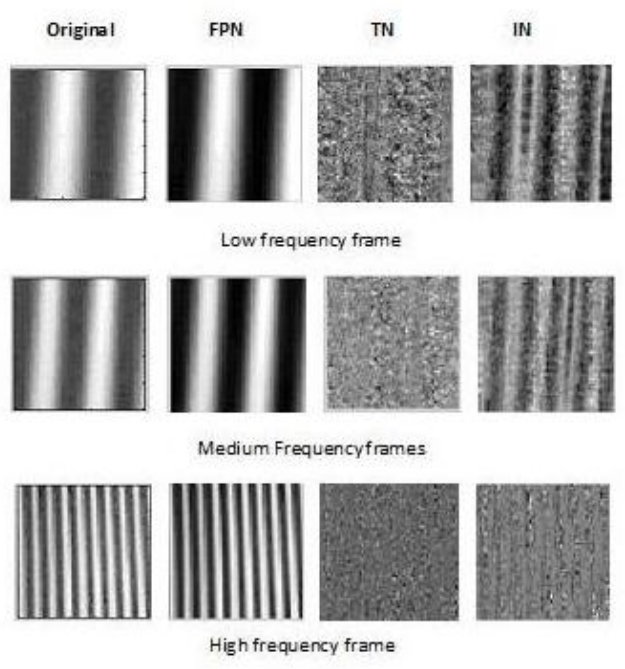

Figure 9: Results using PCA showing the spatial distribution of noises.

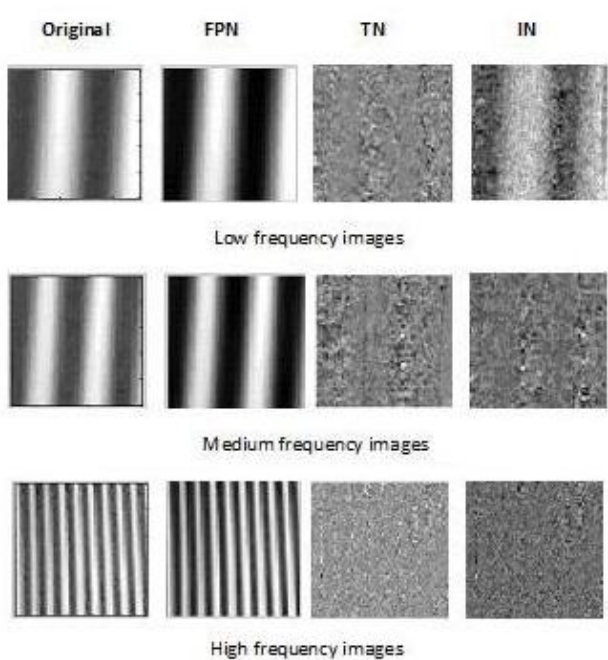

Figure 10: FPN are the results of PCA while IN and TN are extracted from non-FPN images using ICA technique.

\section{Conclusion}

In this paper ICA method has been applied for the characterization of artifacts in fully digital image-acquisition systems. The practical application was performed using video sequences taken 
PCA

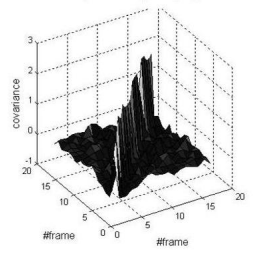

(a)

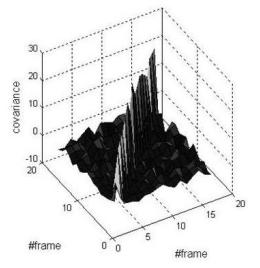

(b)

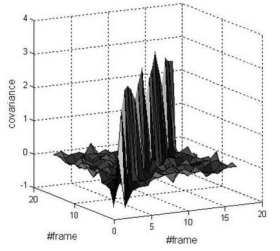

(c)
ICA

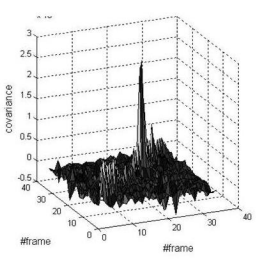

(d)

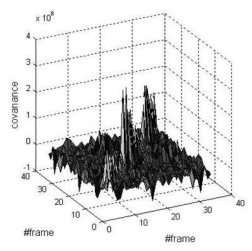

(e)

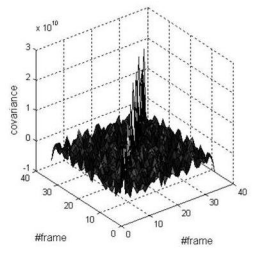

(f)

Figure 11: a) Covariance of TN using Low Frequency. b) Covariance of TN using Medium Frequency. c) Covariance of TN using High Frequency. d) Covariance of TN using Low Frequency. e) Covariance of TN using Medium Frequency. f) Covariance of TN using High Frequency

with USB cameras. PCA was applied as an integral part of ICA. By PCA method two different noise patterns have been attained, the FPN and Non-FPN. The Non-FPN was a mixture of IN and TN noise patterns which are completely independent of each other. Therefore, ICA has been applied to it. The resultant IN and TN from ICA methods are much closer in shape to the actual noise characterizations. One of the major reasons for this is the ability of ICA to extract independent components. This work can be further extended for bad pixel analysis. 

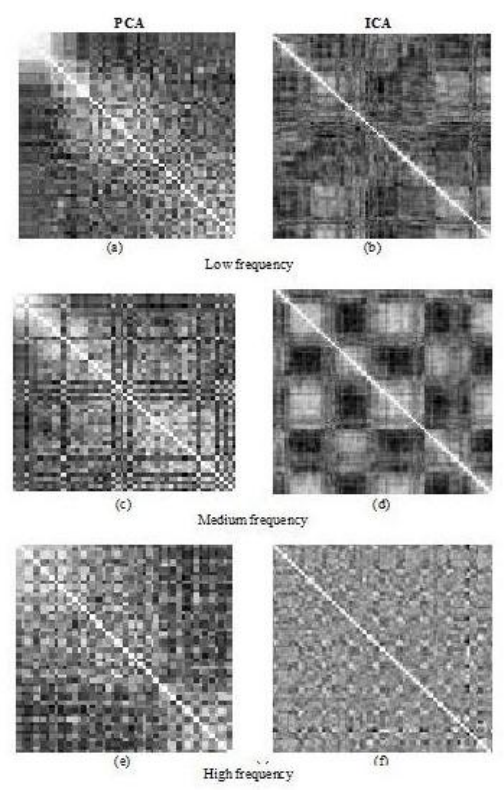

Figure 12: Correlation for IN using PCA in (a), (c), (e) and ICA in (b), (d) and (f).

\section{Bibliography}

[1] Jose Manuel, Lopez-Alonso, and Javier Alda, "Characterization of artfacts in fully digital image-acquisition systems: Application to web cameras,", in Society of Photo-Optical Instrumentation Engineers(SPIE), 2004, vol. 43, pp. 257-265.

[2] Heli T. Hytti, "Characterization of digital image noise properties based on raw data," in Image Quality and System Performance III, 2006, vol. 6059, 60590A.

[3] Tetsuya Takiguchi and Yasuo Ariki, "Pca-based speech enhancement for distorted speech recognition,", in Journal of multimedia, 2005, vol. 2, pp. 13-18.

[4] M. Stewart Bartlett, Javier R. Movellan, and Terrence J. Sejnowski, "Face recognition by independent component analysis,", in IEEE Transactions on Neural Networks,, 2002, pp. $1450-1464$.

[5] Bell A. J., T. J. Sejnowski, and Vision Res, "The independent components of natural scenes are edge filters," in In Neural Comput, 1997, vol. 37, pp. 3327-3338.

[6] S. Makeig, A. J. Bell, T.-P. Jung, and T. J. Sejnowski, "What is the goal of sensory coding?,", in In Neural Comput, 1994, vol. 6, pp. 559-601.

[7] M. J. McKeown, S. Makeig, G. G. Brown, T.-P. Jung, S. S. Kindermann, A. J. Bell, and T. J. Sejnowski, "Analysis of fmri by decomposition into independent spatial components,", in Human Brain Mapping, 1998, vol. 6, pp. 160-188. 